\title{
28 Research Square \\ Schwannoma of Left Chest Wall: a Case Report and Literature Review
}

\section{Congcong Li}

The First Hospital of Jilin University

\section{Ze Tang}

The First Hospital of Jilin University

\section{Da Qin}

The First Hospital of Jilin University

\section{Tianyu Lu}

The First Hospital of Jilin University

\section{Yue Yang}

The First Hospital of Jilin University

Youbin Cui ( $\nabla$ cuiyb@jlu.edu.cn )

The First Hospital of Jilin University https://orcid.org/0000-0002-6682-1376

\section{Case report}

Keywords: Tumor of chest wall, Schwannoma, Solitary fibroma

Posted Date: November 8th, 2021

DOI: https://doi.org/10.21203/rs.3.rs-1013298/v1

License: (c) (1) This work is licensed under a Creative Commons Attribution 4.0 International License. Read Full License 


\section{Abstract}

Background: Schwannoma of chest wall is rare intercostal nerve tumor originated from Schwann cells. Here, we present a rare case about schwannoma was misdiagnosed as solitary fibroma by preoperative biopsy. We intend to improve clinicians' understanding of the disease by discussing the clinical manifestations, diagnostic points and differential diagnosis of patients with thoracic wall schwannoma.

Case presentation: A 38-year-old male presented with "left chest pain for more than 1 month". Enhanced Computer Tomography (CT) can of the lung revealed space occupying lesions in the left lower posterior chest wall. Preoperative biopsy was solitary fibroma. After the improvement of preoperative preparation, the patients were given surgical treatment, postoperative pathology showed: schwannoma of chest wall. Chest pain improved after operation. The patients were followed up for 8 months.

Discussion and Conclusions: Schwannoma, especially in the chest wall, is rare, with atypical clinical symptoms, single imaging manifestations, high variable rate of puncture pathology, and easy to be misdiagnosed. Complete surgical resection of tumor is the main treatment, and no new adjuvant / adjuvant therapy has been reported yet.

\section{Background}

Thoracic wall schwannoma is a rare intercostal nerve tumor originated from intercostal Schwann cells. We reported a case of solitary fibroma highly suspected by preoperative enhanced CT and confirmed by preoperative biopsy, but diagnosed as schwannoma of chest wall by postoperative pathology in Department of thoracic surgery, The First Bethune Hospital of Jilin University.

This case reports the thoracic wall schwannoma was misdiagnosed as solitary fibroma with progressive chest pain and successfully treated with minimally invasive video-assisted thoracoscopic surgery.

\section{Case Presentation}

A 38-year-old male with left chest pain for one month was admitted to Beijing chest hospital on June 20, 2020. Lung CT showed that the left lower posterior thoracic cavity space occupying lesion, adjacent ribs were involved, which was larger than one year ago, the lesion may origin from pleural or neurogenic lesions. Then the patient underwent percutaneous tumor puncture in Beijing hospital on July 10, 2020. Pathological diagnosis: a small amount of spindle cell tumor tissue, combined with immunohistochemistry, was consistent with solitary fibrous tumor. Immunohistochemical results showed: ck1/3(-), ck8(-), S-100(+++), NF(-), Ki-67(8\%+), CD34 (partial+),Vimentin(+++), Ventara ALK(D5F3) $(-), \operatorname{SMA}(-)$, Desmin(-), CD163(+++), CD34(-), SOX10(+++), STAT6(+), EMA(-), P53(20\%+, wild type), CD35(-), CD21(+). The patient sought medical advice in our hospital from July 21, 2020. When admitted, the left chest pain was as before, without any discomfort such as dyspnea, chest tightness, shortness of breath, cough, expectoration, abdominal pain and diarrhea, dizziness and headache, and denied symptoms such as low fever, fatigue, sweating and other symptoms, and the vital signs were stable. 
Deny the history of hypertension, diabetes, heart disease, family history and tuberculosis history. A systemic physical examination was unremarkable. Auxiliary inspections including blood routine, urine routine, coagulation routine, liver function, renal function, ion, Erythrocyte sedimentation rate (ESR), tumor markers were normal, tuberculosis antibody, sputum smear and t-spot were negative. A chest enhanced CT demonstrated massive high-density shadow protruding into the thoracic cavity was seen on the left lower posterior chest wall, with the size of about $7.4 \mathrm{~cm} * 5.2 \mathrm{~cm} * 5.5 \mathrm{~cm}$, regular shape, smooth edge, and wide base connected with the left pleura. The arterial phase: $23 \mathrm{HU}$, venous phase: $30 \mathrm{HU}$, with small vascular shadow in it, considering solitary fibroma; there was no obvious enlarged lymph node shadow in mediastinum (Figure 1).

Figure 1D is the three-dimensional reconstruction figure of chest wall mass based on chest CT images, after preoperative preparation was improved, the operation was started. The anesthesia of double lumen endotracheal intubation came into effect, a tumor with the size of $7 \mathrm{~cm} * 6 \mathrm{~cm} * 6 \mathrm{~cm}$ was found between the 7th and 8th ribs of the left chest wall under three dimensional (3D) video-assisted thoracoscopy. Then the tumor was completely removed (Figure 2). Intraoperative rapid pathology reported: interlobar tumor of chest wall. Postoperative histopathological analysis showed that the tumor is consistent with schwannoma (Figure 3), on immunohistochemistry, S-100 protein and Vimentin are positive (Figure 4). The patient recovered well after operation, discharged 5 days after operation, followed up by telephone 8 months after operation, the patient's chest pain relived, and life as usual.

\section{Discussion And Conclusions}

Primary chest wall benign tumor is not common, patients often were found with chest wall tumor because of persistent chest wall pain(1). Chest wall schwannoma, which is seldom seen, originates from Schwann cells of the nerve sheath(2). It is a lobulated or spherical solitary, encapsulated tumor that grows slowly in the retromediastinal pleural cavity $(3,4)$. Schwannoma accounts for about $5 \%$ of all benign soft tissue tumors; it is most common in spinal cord and nerve root, protruding from paravertebral to mediastinum, and rarely in chest wall. The incidence rate is almost no gender difference, almost between 20 years old and 30 years old. Pain and nervous system symptoms are almost absent in small tumors $(<5 \mathrm{~cm})(5,6)$.

Isolated fibromas are mainly derived from a CD34 positive dendritic mesenchymal cell and belong to a kind of interlobular tumor(7), differentiating into myofibroblasts and fibroblasts is characteristic of isolated fibroma. Solitary fibroma is rare, most of them are benign, about $12 \%-20 \%$ of them have local recurrence, distant metastasis and other malignant changes after several years of stability, and eventually lead to death $(8,9)$. The main age of onset was 60 to 70 years old(10). Incidence rate of male and female was similar(11).

Neurilemmoma and solitary fibroma grow slowly and most of them are asymptomatic. They are only found when the tumor growth oppresses the surrounding tissue and produces pain, cough and other 
symptoms. It is difficult to distinguish them by clinical symptoms (Table 1), CT showed they are clear and homogeneous soft tissue tumors(11-13).

Pathologically, schwannoma was composed of Antoni type A and Antoni type B. the tumor was encapsulated without axons, and there might be blood vessels in the tumor, the specific immunohistochemical features of schwannoma including S-100 protein, vimentin and enolase were positive, while neurofilament protein was negative $(10,14,15)$. In particular, S-100 protein can be seen in almost all schwannomas and is a marker of benign schwannomas $(15,16)$. Solitary fibroma presented as spindle cell tumor pathologically(17), immunohistochemical features were positive for CD34,vimentin, $B$ cell lymphoma-2(bcl-2) or CD99 $(10,11,18)$, vimentin is a marker of mesenchymal cells, while CD34 as the most consistent conventional marker, is a transmembrane glycoprotein that widely exists in dendritic mesenchymal cells and participates in antigen presentation $(11,18)$. Recent oncological studies indicate that activator of transcription 6 (STAT 6) antibody is an immunohistochemical marker for solitary fibroma(19). However, keratin, Desmin, S-100 protein, carcinoembryonic antigen, a 1-ACT and F-VIII related antigen were negative $(18,20)$.

The patient's clinical symptoms have no obvious characteristics, imaging studies are difficult to differentiate from other tumors, and puncture pathology shows that CD34 and vimentin are positive, suggesting that they are derived from mesenchymal cells. The puncture tissue is small and can't show the whole picture of the tumor, and there are differences in tumor cells. Therefore, it is misdiagnosed as solitary neurofibroma before operation. Because of the similar imaging findings and not prominent clinical manifestations, it is a great challenge to differentiate schwannoma from solitary fibroma. Key points of differentiation between schwannoma and solitary fibroma are listed in Table 1. 
Table 1

key points of differentiation between schwannoma and solitary fibroma

\section{Thoracic schwannoma}

Origion nerve sheath schwannoma

Lesion spinal cord and nerve root

location

Incidence seldom seen

rate

Oneset age aged 20 to 30 Years

Morbidity of similar

male and

female

Malignant low

rate

CT a well-defined, rounded, soft tissue

performance

density lesion, isoattenuation or mild

hypoattenuation to the chest wall muscle

on unenhanced CT(21)

Pathological

features
S-100 protein, vimentin and enolases are positive,neurofilament protein is negative solitary fibroma

CD34 positive dendritic mesenchymal cell

visceral interpleural cortex

seldom seen

aged 60 to 70 Years

similar

high (12-20\%)

Great variation: ovoid or elliptical, solitary, the "geographic pattern" of a rich vascularization of the tumor, necrosis or calcifications(22)

STAT6, CD34, CD99 or bcl-2 are positive

Schwannoma, especially in the chest wall, is rare. The clinical symptoms are not obvious or even without clinical manifestations. The imaging manifestations are single, and the puncture pathology variable rate is high. Further histopathological and immunohistochemical examination are needed to make a definite diagnosis. Therefore, there is a view that preoperative biopsy will not bring benefits to patients, and does not change the outcome of disease treatment, so it is recommended that direct surgical treatment. Although schwannoma is a kind of benign peripheral schwannoma, it still has the possibility of malignant transformation. At present, the choice of diagnosis and treatment is still based on complete surgical resection of the tumor, and no neoadjuvant / adjuvant treatment has been reported.

\section{Abbreviations}

CT: Computer Tomography; 3D: three dimensional; ESR: Erythrocyte sedimentation rate; ck: cytokeratin; S100: Soluble protein-100; NF: Neurofilament protein; Ki-67: Antigen Ki-67; CD: Recombinant Cluster Of Differentiation; ALK: Anaplastic lymphoma kinase; SMA: Smooth Muscle Actin; SOX10: SRY-related HMGbox 10 protein; STAT6: Signal Transducer and Activator of Transcription 6; EMA: Epithelial Membranes Antigen; P53: Protein 53 


\section{Declarations}

\section{Ethics approval and consent to participate}

Not applicable.

\section{Consent for publication}

Informed consent was obtained from the patient.

\section{Availability of data and materials}

All data generated or analysed during this study are included in this published article.

\section{Competing interests}

The Authors declare that there are no conflicts of interest.

\section{Funding}

Funding information is not available.

\section{Authors' contributions}

Author Youbin Cui is the main performers of the operation, author Tianyu Lu and Ze Tang are assistants in the surgery, author Yue Yang and Da Qin are in charge of the postoperative recovery of patients, author Congcong $\mathrm{Li}$ and Ze Tang are responsible for article writing and image procession.

\section{Acknowledgements}

Not applicable.

\section{References}

1. Stelzer P, Gay WA, Jr. Tumors of the chest wall. Surg Clin North Am. 1980;60(4):779-91.

2. Albert P, Patel J, Badawy K, Weissinger W, Brenner M, Bourhill I, et al. Peripheral Nerve Schwannoma: A Review of Varying Clinical Presentations and Imaging Findings. J Foot Ankle Surg. 2017;56(3):6327.

3. Ilgan S, Dikmen E, Cetinkanat CG, Dakak M, Güngör A. Schwannomatozis of the Chest Wall: FDG PET Findings. Mol Imaging Radionucl Ther. 2014;23(2):64-6.

4. Galukande M, Khingi A. Chest wall schwannoma presenting as a solitary malignant lesion: a case report. Springerplus. 2016;5(1):1549.

5. Murphey MD, Smith WS, Smith SE, Kransdorf MJ, Temple HT. From the archives of the AFIP. Imaging of musculoskeletal neurogenic tumors: radiologic-pathologic correlation. Radiographics. 
1999;19(5):1253-80.

6. Krishnamurthy A, Raghunandhan GC, Majhi U. Dumbbell Shaped Schwannoma of the Lateral Chest Wall masquandering as a soft tissue sarcoma. Indian J Surg Oncol. 2015;6(3):307-10.

7. Mordenti P, Di Cicilia R, Delfanti R, Capelli P, Paties C, Cavanna L. Solitary fibrous tumors of the pleura: a case report and review of the literature. Tumori. 2013;99(4):e177-83.

8. Briselli M, Mark EJ, Dickersin GR. Solitary fibrous tumors of the pleura: eight new cases and review of 360 cases in the literature. Cancer. 1981;47(11):2678-89.

9. Shanahan B, Redmond KC. Largest known malignant solitary fibrous tumour of the pleura-extended resection warranting cardiopulmonary bypass support. Ir J Med Sci. 2019;188(2):433-5.

10. Dango S, Kirschbaum B, Passlick B. [Solitary fibroma of the pleura - clinical findings and prognosis]. Zentralbl Chir. 2008;133(3):227-30.

11. Abu Arab W. Solitary fibrous tumours of the pleura. Eur J Cardiothorac Surg. 2012;41(3):587-97.

12. Rena O, Filosso PL, Papalia E, Molinatti M, Di Marzio P, Maggi G, et al. Solitary fibrous tumour of the pleura: surgical treatment. Eur J Cardiothorac Surg. 2001;19(2):185-9.

13. Mullan CP, Madan R, Trotman-Dickenson B, Qian X, Jacobson FL, Hunsaker A. Radiology of chest wall masses. AJR Am J Roentgenol. 2011;197(3):W460-70.

14. Ampollini L, Ventura L, Gnetti L, Carbognani P, Silini EM, Rusca M. Giant intercostal nerve schwannoma in a patient with neurofibromatosis type 2. Tumori. 2018;104(6):Np17-np21.

15. Henn LA, Gonzaga RV, Crestani J, Cerski MR. [Intercostal schwannoma simulating pulmonary neoplasms]. Rev Assoc Med Bras (1992). 1998;44(2):146-8.

16. Fanburg-Smith JC, Majidi M, Miettinen M. Keratin expression in schwannoma; a study of 115 retroperitoneal and 22 peripheral schwannomas. Mod Pathol. 2006;19(1):115-21.

17. Tan JH, Hsu AA. Challenges in diagnosis and management of giant solitary fibrous tumour of pleura: a case report. BMC Pulm Med. 2016;16(1):114.

18. Chang YL, Lee YC, Wu CT. Thoracic solitary fibrous tumor: clinical and pathological diversity. Lung Cancer. 1999;23(1):53-60.

19. Davanzo B, Emerson RE, Lisy M, Koniaris LG, Kays JK. Solitary fibrous tumor. Transl Gastroenterol Hepatol. 2018;3:94.

20. Chen L, Sang Y, Zhang Z, Yang W, Chen Y. Strategy for initial en bloc resection of a giant mediastinal solitary fibrous tumor: Judicious usage of cardiopulmonary bypass. Thorac Cancer. 2020;11(7):2048-50.

21. Hu S, Chen Y, Wang Y, Chen KM, Song Q. Clinical and CT manifestation of pleural schwannoma. Acta Radiol. 2012;53(10):1137-41.

22. Savu C, Melinte A, Posea R, Galie N, Balescu I, Diaconu C, et al. Pleural Solitary Fibrous Tumors-A Retrospective Study on 45 Patients. Medicina (Kaunas). 2020;56(4).

\section{Figures}




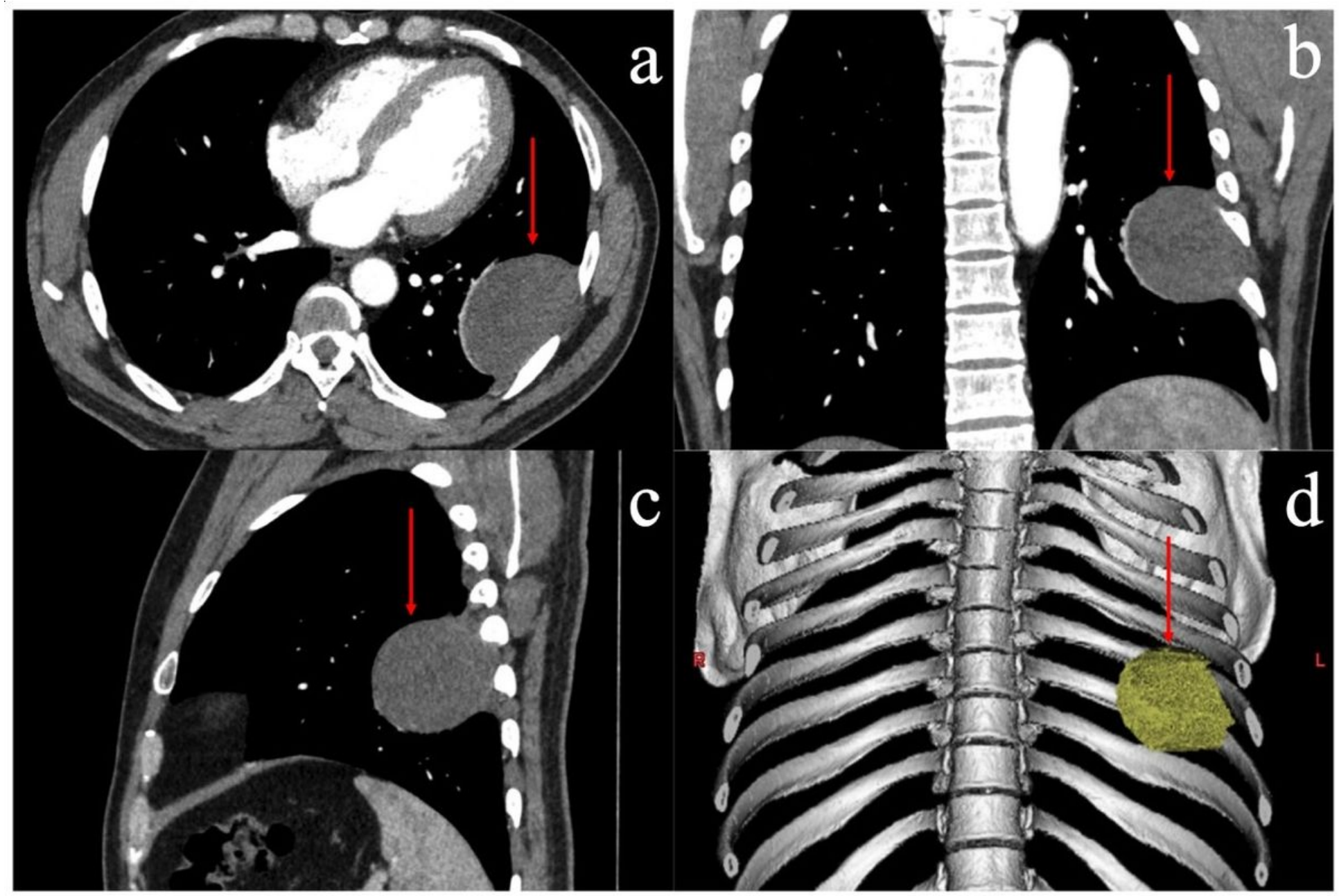

Figure 1

Chest enhanced CT. Figure 1A-1C 『chest enhanced CT showed the tumor (narrow) of the left inferior posterior wall protrudes into the thoracic cavity with regular shape and smooth edge. The broad base is connected with the left pleura, and the shadow of small blood vessels can be seen in it. Figure 1D囚threedimensional reconstruction figure of chest wall mass based on chest CT images. 


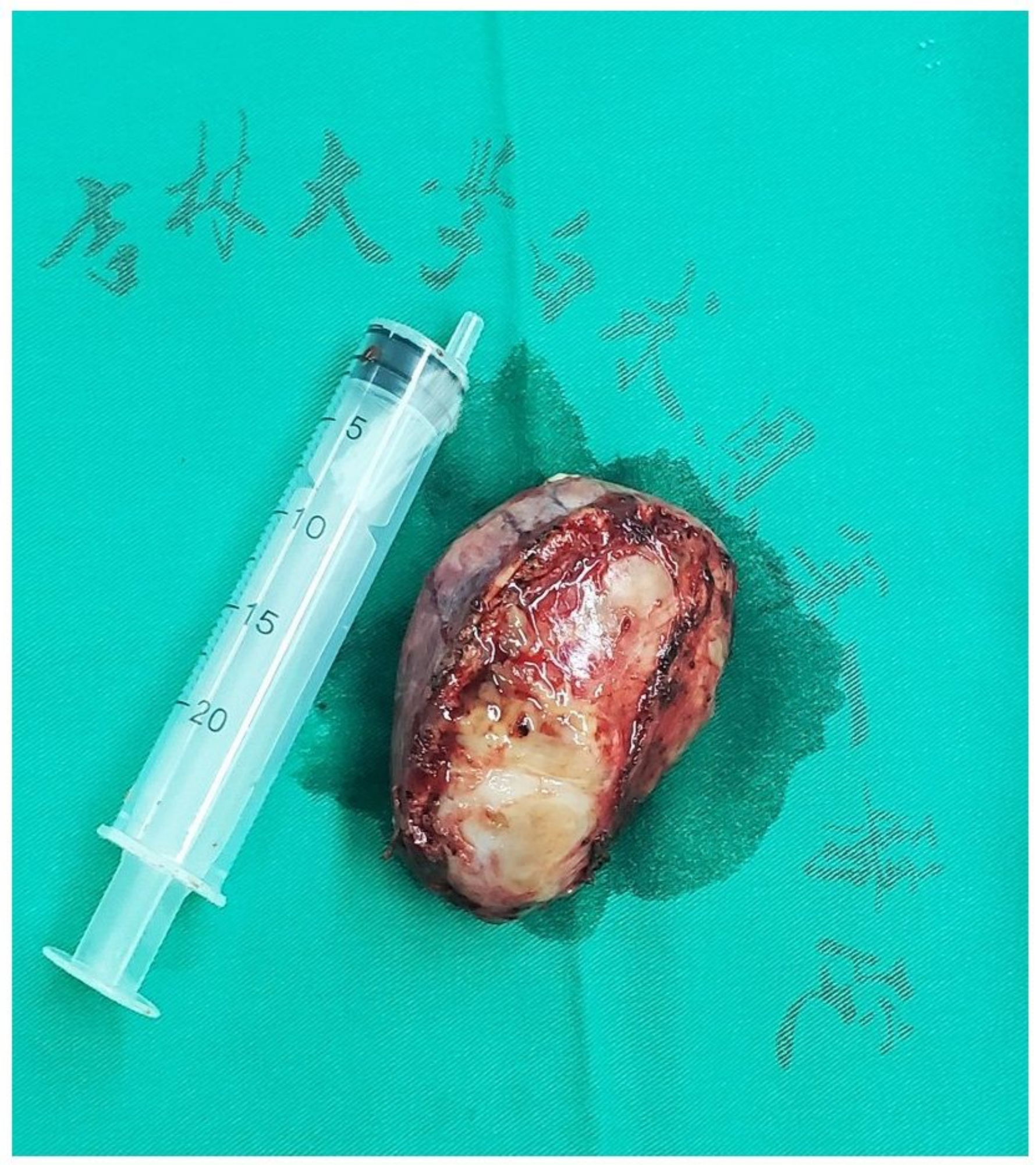

Figure 2

Gross specimen exhibits the size of the chest wall tumor is about $7.4 \mathrm{~cm} * 5.2 \mathrm{~cm} * 5.5 \mathrm{~cm}$, which is tough and has smooth diolame. 


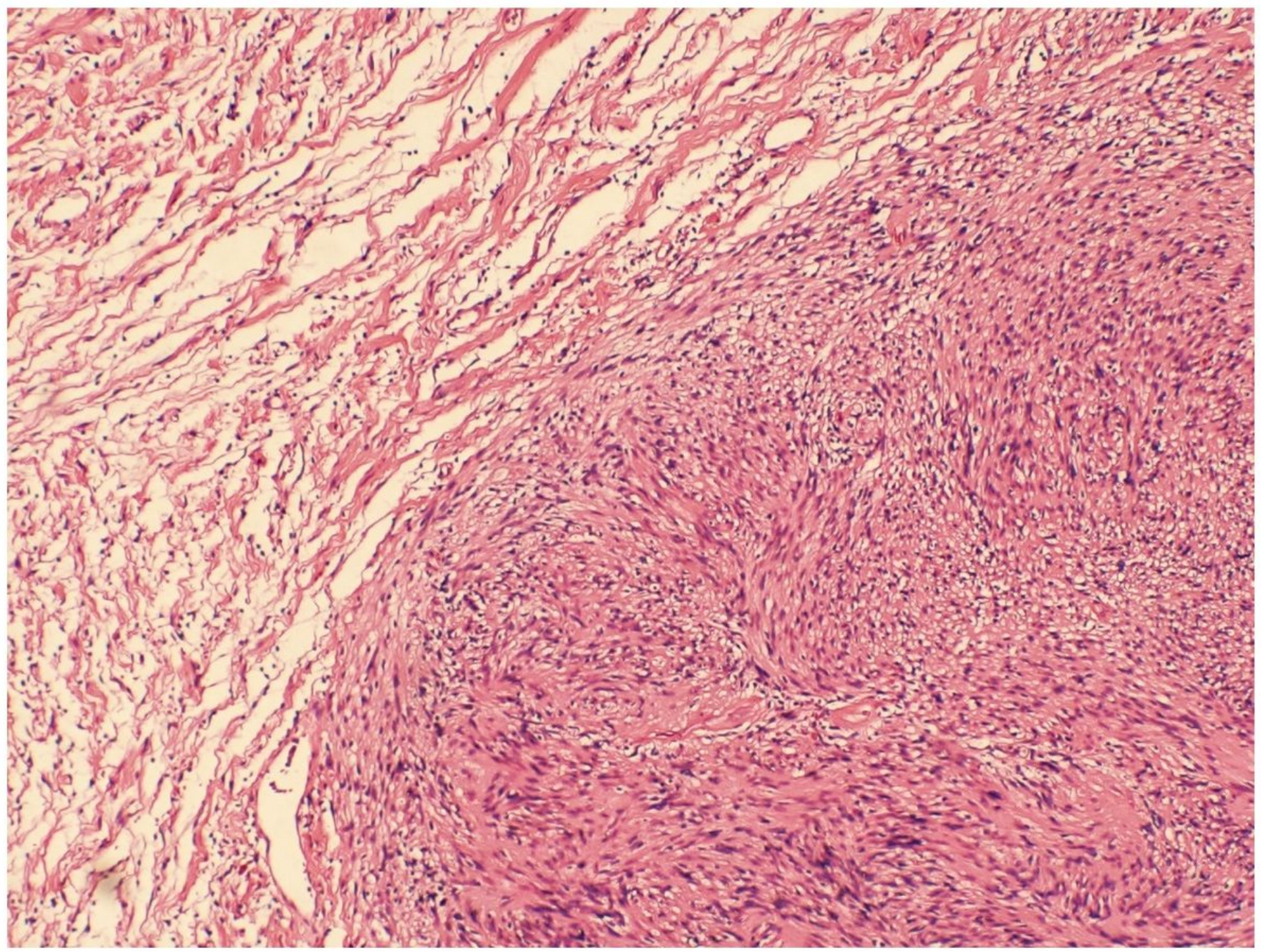

\section{Figure 3}

H\&E staining demonstrates the cell morphology was consistent with that of schwann.

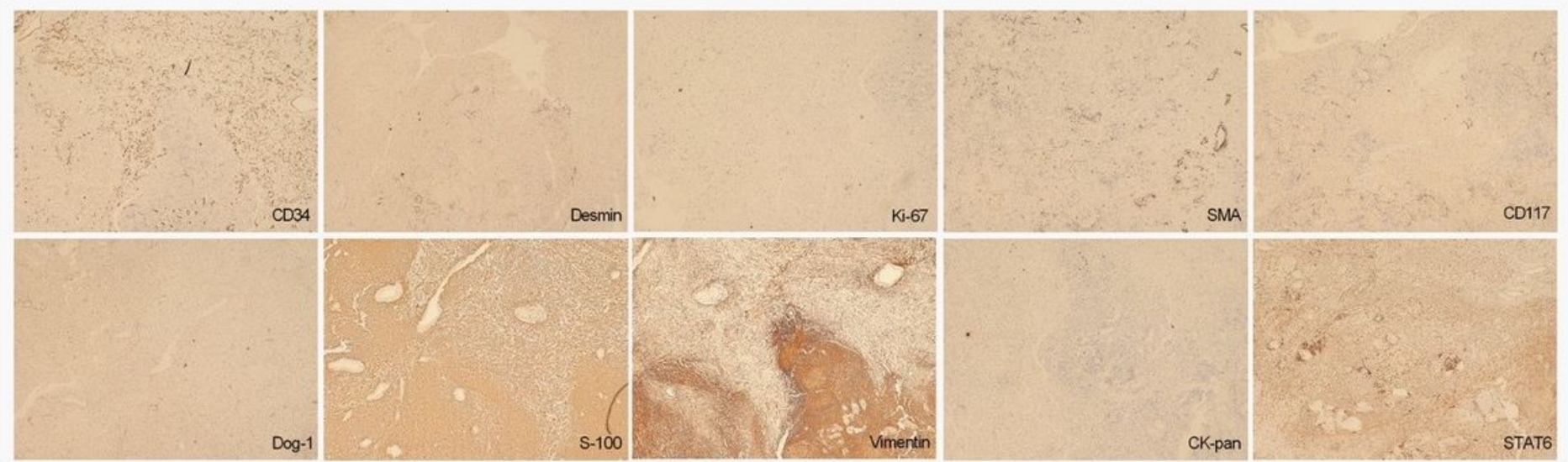

Figure 4 
Immunohistochemical analysis: CD34 (Partial positive), Desmin (-), Ki-67 (+5\%), SMA (-), CD117 (-), Dog-1 $(-)$, S-100 (+), Vimentin (+), CK-pan (-), STAT6 (-).

\section{Supplementary Files}

This is a list of supplementary files associated with this preprint. Click to download.

- CAREchecklist.pdf 